\title{
The Media pluralism monitor: Conceptualizing media pluralism for the online environment
}

\author{
Elda Brogi
}

How to cite this article:

Brogi, Elda (2020). "The media pluralism monitor: Conceptualizing media pluralism for the online environment". Profesional de la información, v. 29, n. 5, e290529.

https://doi.org/10.3145/epi.2020.sep.29

Invited manuscript received on $18^{\text {th }}$ October 2020

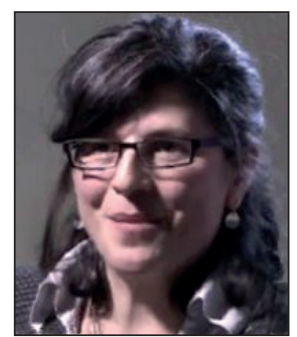

\author{
Elda Brogi \\ https://orcid.org/0000-0003-2682-0528 \\ Centre for Media Pluralism and Media Freedom \\ European University Institute \\ Via Boccaccio, 121/111 \\ 50133 Florence, Italy \\ elda.brogi@eui.eu
}

\begin{abstract}
Media pluralism is an essential feature and pillar of contemporary democracies. It is a corollary of the right to freedom of expression, as guaranteed by European national constitutions, as well as by the Convention for the protection of human rights and fundamental freedoms (art. 10) and by the Charter of fundamental rights of the European Union (art. 11). Ensuring a plural media environment is a precondition for democracy and may be seen as a policy goal. The availability and accessibility of diverse information and views create the conditions for citizens to form and express their opinions and participate in the democratic debate in an informed way. Due to technological developments and the rapid rise of digital platforms intermediating online content, the concept of media pluralism has been reinterpreted at both academic and policy level, taking into account the recent structural changes in the media sector. The Media pluralism monitor is a methodology to assess the risks to media pluralism, based on a set of 20 indicators covering a broad definition of media pluralism and, therefore, having a holistic perspective. The recent implementations of the tool have taken into account in particular the risks stemming from the online environment while maintaining a holistic approach to conceptualizing standards and benchmarks for the indicators.
\end{abstract}

\section{Keywords}

Media pluralism; Media freedom; Media pluralism monitor; Media; Exposure diversity; Digital platforms; Digital dominance; Rule of law; Europe.

\section{Introduction}

The importance of ensuring a plural media environment is acknowledged by all contemporary democracies and is a shared policy goal. Freedom of expression and its corollaries of freedom of the media and media pluralism are considered cornerstones of the rule of law and preconditions for a sound political debate. As stated in Recommendation 1 (2018) of the Council of Europe on media pluralism and transparency of media ownership, they

"ensure the availability and accessibility of diverse information and views, on the basis of which individuals can form and express their opinions and exchange information and ideas."

While it is widely agreed amongst academics and policymakers that media pluralism is a democratic value, essential for the integrity of the democratic discourse and procedures, the definition of media pluralism in itself is quite widely debated and influenced by different political, economic, and legal contexts, by the academic approach, and by market and technological developments. The concept of media pluralism can be elaborated based on different meanings that range from the "marketplace of ideas" (Mill, 1859) of political liberalism, to a definition based on the notion of the "public sphere" (Habermas, 1990). According to the latter notion, which has become a feature of the European debate on this 
topic, media pluralism is associated with the definition of deliberative democracy and implies that citizens have access to a wide array of information as a precondition to be able to participate in the democratic debate.

Media pluralism has been interpreted based on many different nuances. Media pluralism has been conceived as a representation of geographical and cultural diversity, as well as a space reserved for minority groups. It is also meant as a feature of public media, whose remit is to provide plural information in the public interest. In many contexts, the importance of media pluralism has been acknowledged only during electoral periods, in which case the concept of media pluralism is strictly connected with ensuring that all the candidates and parties have equal and fair media access conditions. This is needed to satisfy both the requirement for a fair electoral campaign and to ensure that voters receive information from all the parties competing in the elections.

Amongst all the interpretations of the notion of media pluralism, that which focuses on diversity and transparency of media ownership has been the most widely exploited by policymakers. For many years and many authors, media pluralism has meant just (external) plurality of ownership, where concentration in the media market, or even a potential concentration in a market that naturally evolves towards oligopoly or monopoly, has been seen as a risk to the democratic debate by potentially limiting the diversity of voices offered by the market itself. In parallel, the transparency of media ownership has acquired importance as an instrument, on the one hand to evaluate the concentration of the market and, on the other, to reveal the vested interests of media owners and the potential bias in the editorial line of a media outlet.

More recently, the notion of media pluralism in its traditional meaning has been, nonetheless, challenged as a principle and a policy goal, also due to technological developments. Karppinen (2013), for instance, deconstructing the established interpretation of the concept of media pluralism, associated the term with the notion of "radical pluralism" as the "continuous contestation" of media power relations. In this perspective, pluralism is seen as the capacity of different groups of media actors to challenge the established hegemonic media order and provide alternatives.

Defining media pluralism in the age of new media services, the Internet, the World Wide Web, and social media has proved truly challenging for scholars and policymakers. While information abundance sparked initial optimism as new technologies allowed cheap and universal systems to disseminate any kind of information, the consolidation of (big) companies as intermediaries of the information itself, i.e., the way in which these platforms operate as gatekeepers of online information, has sparked criticism on how the digital environment can be effectively open and plural, and whether the democratic discourse really benefits from this (Moore; Tambini, 2018; Parcu, 2019).

Against this background, many authors and policymakers have engaged in a reinterpretation of the sense and meaning of media pluralism. For instance, the most recent academic and policy debate has stressed the importance of defining plurality, starting from an analysis of the condition of users within the new media ecosystem; search engines, social networks, apps, and nonlinear audiovisual services are the new gatekeepers of accessing general information, especially information in the public interest. In an online environment that increasingly relies on personalized news recommendation outlets and personalized information "bubbles," users may be less exposed to a diversity of content. Citizens tend to be exposed mainly to content which reinforces or confirms their previously formed views (so-called filter bubbles or echo-chambers) (Parisier, 2012), while they are progressively ${ }^{1}$ less exposed to content in the general public interest or, in any case, content that is relevant for critical participation in democratic life. Based on these assumptions, the notion of "exposure diversity" (Helberger, 2018) is becoming more relevant in the formation of a new definition for media pluralism; exposure to different voices is no longer linked to the number of media outlets provided by the market but to how algorithms can be designed to expose the citizen to more diverse content and, in particular, to "public interest" rather than just popular or personalized content.

This brings us to another terminology conundrum: the definition of "media" itself is currently another element of debate amongst scholars and policymakers. Within the new digital landscape, "mass media" is being progressively replaced by a system of one-to-one communication, relying on narrowcasting, i.e., content provided on-demand and information received based on profiling. Theories and conceptualizations on "media pluralism" stem from the need to respond to a mass media environment/market, potentially concentrated on or catering to requests for diversity within traditional press and broadcasters. The genesis of the notion of "media pluralism" is therefore associated with a print and linear broadcasting environment that no longer exists as such. Instead, the "new media" environment is characterized by a segmentation of the audience, the proliferation of one-to-one personalized information services, and an algorithmic-driven communication premise based on profiling. In the recent past, the clear distinction between mass media and personal communication has been one of the pillars on which different regulations and policies, including on pluralism, have been established. These were based on a clear difference between the impact of a (wide) "public at large" communication and the (limited) impact that personal communication, i.e., with specific and limited "recipients," has on public opinion. In the contemporary information ecosystem, the boundaries between "mass media" and "personal communication" are no longer sufficiently clear. While it is widely acknowledged that plural information is what is needed to ensure the integrity of democracy, the personalization of messages delivered to a wide audience has posed a

The MPM is a research tool that was designed to identify and measure potential risks to media pluralism in the member states of the European Union 
new dilemma over what type of communication can be considered relevant to public discourse, and what should be taken into account when evaluating the level of pluralism of the digital media environment.

\section{The Media pluralism monitor: An operative and "European" notion of media pluralism}

After many years of implementation, the experience of the Media pluralism monitor (MPM) project can probably be used as a point of reference for defining media pluralism in the digital age. The MPM is a research tool that was designed to identify and measure potential risks to media pluralism in the member states of the European Union. Its genesis dates back to 2009 with the publication of the independent study on Indicators for media pluralism in the member states-Towards a risk-based approach (KU Leuven, 2009), aimed at defining a set of indicators and a methodology that could be useful in "measuring" threats to pluralism in the member states. Using this as a matrix and to inspire their methodology, the Centre for Media Pluralism and Media Freedom at the European University Institute has updated, operationalized, and implemented the Media pluralism monitor in EU member states and candidate countries. Since then, the MPM tool has been constantly revised and refined before each implementation to cope better with the changing digital environment and keep it up to date with new technological developments.

The peculiarity of the MPM is that it does not prefer a notion of media pluralism; instead, it builds on the different national and European traditions and definitions to elaborate a set of indicators that tend to cover all possible aspects involved in the definition of media pluralism in a broad European sense. The instrument is based on standards that are widely shared amongst member states of the European Union, as elaborated by the Council of Europe and the European Commission, and relies on constitutional traditions common to the EU member states. It relies on a broad definition of media pluralism that entails legal, economic, and sociopolitical aspects. It therefore takes a holistic approach that considers all the different nuances of the definition of media pluralism. Indeed, the MPM organizes the risks in media pluralism into four main areas:

- basic protection,

- market plurality,

- political independence, and

- social inclusivity.

This allows for an assessment that covers the different components and meanings of "media pluralism," as developed by academia and policymakers. These four areas are assessed according to the scoring of 20 indicators and 200 variables in total. The variables are clustered into sub-indicators that cover a broad notion of media pluralism encompassing political, cultural, geographical, structural, and content-related dimensions.

Table 1. Areas and indicators covered by the MPM2020

\begin{tabular}{|c|c|c|c|}
\hline Basic protection & Market plurality & Political independence & Social inclusiveness \\
\hline $\begin{array}{l}\text { Protection of freedom of expres- } \\
\text { sion }\end{array}$ & Transparency of media ownership & Political independence of media & Access to media for minorities \\
\hline Protection of right to information & News media concentration & Editorial autonomy & $\begin{array}{l}\text { Access to media for local / } \\
\text { regional communities and for } \\
\text { community media }\end{array}$ \\
\hline $\begin{array}{l}\text { Journalistic profession, standards } \\
\text { and protection }\end{array}$ & $\begin{array}{l}\text { Online platforms concentration } \\
\text { and competition enforcement }\end{array}$ & $\begin{array}{l}\text { Audiovisual media, online platfor- } \\
\text { ms and elections }\end{array}$ & $\begin{array}{l}\text { Access to media for people with } \\
\text { disabilities }\end{array}$ \\
\hline $\begin{array}{l}\text { Independence and effectiveness } \\
\text { of the media authority }\end{array}$ & Media viability & $\begin{array}{l}\text { State regulation of resources and } \\
\text { support to media sector }\end{array}$ & Access to media for women \\
\hline $\begin{array}{l}\text { Universal reach of traditional me- } \\
\text { dia and access to the internet }\end{array}$ & $\begin{array}{l}\text { Commercial \& owner influence } \\
\text { over editorial content }\end{array}$ & $\begin{array}{l}\text { Public service media governance } \\
\text { and funding }\end{array}$ & Media literacy \\
\hline
\end{tabular}

Data for the MPM are collected by a network of teams at national level through a structured questionnaire. This method allows for the gathering of both quantitative and qualitative data. Additionally, the MPM method allows for the quantitative analysis of answers and the production of a numerical risk assessment, which is important to obtain results that are comparable across countries. A score of 0 is attributed to variables that present a low risk, 0.5 to variables that present medium risk, and 1 to variables that present a high risk. The final assessment per area of risk is carried out using a specific algorithm that was developed by the CMPF, based on which risk assessment is calculated as the average of the average score of variables of the same type. The final results are risk assessments per sub-indicator, indicator, and area. This allows for a balanced score combining legal, economic, and factual data. Another interesting feature of the MPM is that the evaluations are a result of a combination of assessments that measure compliance to normative standards from a formal point of view (for

The CMPF has always considered "news and current affairs" as the core of the MPM analysis, focusing mostly on the type of content considered relevant in regards to public interest, instead of on the specific type of media used to distribute the information 
example, the existence of legal standards that comply with European or international standards on paper) and the reality of the implementation of the law, the economic structure, and the sociopolitical situation.

As the MPM project runs periodically, to meet the challenges emerging from this large-scale comparative analysis, it mostly relies on secondary data collected by national experts. These data are supplemented with primary sources gathered through interviews and document analyses (e.g., of legal and academic texts), together with the involvement of a "group of experts" to evaluate the variables that are difficult to measure, require a qualitative type of measurement, and/or lack measurable and easily verifiable data.

Being implemented several times since 2014, the MPM's geographical reach and holistic approach make it a useful and unique research methodology, as it collects a dataset based on normative standards. Covering different national cases, the results should therefore be interpreted based on political, economic, and national contexts, and the dataset can also be effectively used as a comparative instrument. Furthermore, it also provides relevance to some benchmarks that should be taken into account by policymakers at national level when defining policies to foster media pluralism itself. The methodology developed by the MPM project proved to be very effective in determining different benchmarks that constitute the building blocks of the assessment of a broad and holistic notion of pluralism, which includes an analysis of the respect of fundamental rights and competition in the media market, thereby guaranteeing fair political discourse and inclusion.

The recent developments and fine-tuning of the MPM tool are particularly relevant as they try to develop new standards and benchmarks for the new media environment with the intention of accommodating the complexity of technological evolution, as well as adjusting to the dimension of exposure diversity. The MPM tool has been developed and refined over the years keeping in mind the dramatic changes in the EU and international media landscape, as well as strong disruptions due to technological developments.

Consequently, there have been two predominant new challenges for the MPM tool since it was first operationalized. The first was to define the scope of the MPM tool, taking into consideration the difficult definition of "media" within the new digital environment, i.e., with all the new forms of personalized information. The second was to evaluate whether or not the normative standards used to assess media pluralism could be reproposed for the new media environment, given the specificities and ontological differences between the "old" and "new" means of information and communication.

The approaches of the Centre for Media Pluralism and Media Freedom in this regard have been very practical, as well as both conservative and innovative. In terms of the scope of the MPM tool, the Centre has always considered "news and current affairs" as the core of the MPM analysis, focusing mostly on the type of content considered relevant in regards to public interest, instead of on the specific type of media used to distribute the information. This is a practical solution that has proved useful for the inclusion of all the media that have an impact on public opinion in the MPM analysis, regardless of the way in which they are distributed or whether they are considered to be based on the traditional notion of "mass media." Therefore, the evolving definition of media can be taken into account, or better included, in the scope of the assessment of all the various services, both online and offline, that offer news and information on current affairs and that, in the end, contribute to the shaping of "public opinion," regardless of the means of distribution. Therefore, as in previous revisions of the MPM, the scope of the analysis covers "news and current affairs" more than specific kinds of media. In this way, the MPM covers all media (from an etymological standpoint) conducive to the public debate. As a consequence, the analysis and scope of the MPM are not limited to mass media but include all new forms of targeted, algorithmically driven communication, insofar as they can be considered relevant to the shaping of public opinion or interfering in the democratic process.

In terms of the proposed normative standards, the CMPF began with the widely shared normative assumption that freedom and pluralism of media are core principles and values that make up the essential foundations of contemporary democracy. They are central to the functioning of a democratic society as they help to ensure the availability and accessibility of diverse information and views, based on which individuals can form and express their opinions and exchange information and ideas. ${ }^{3}$ The "conservative" approach of the CMPF was to consider the actual and logical normative standards that had been already developed in the MPM when addressing online phenomena, but -and this is the innovative part- to reshape the normative standards and indicators based on a new conceptualization of the principles they entail. The reconceptualization of the normative standards also implies the reconsideration and reconceptualization of the new types of risks that come with the new characteristics of the online environment. For instance, transparency is a principle that turned out to be paramount in the analysis of "digital pluralism." This standard has been reinterpreted and applied not only in the light of freedom of information and media ownership but also as a desirable normative standard for freedom of expression online, considering the ambiguity within content moderation and curation practices online. Likewise, transparency is an element that is relevant in determining the fairness of an electoral campaign and the resources devoted to electoral advertising online by political parties. The standard of 
transparency was extended to a wider list of indicators in the MPM (i.e., it was not limited to ownership and administration transparency for the purpose of access to information) and broadly interpreted, with the inclusion of accountability of online platforms in terms of content moderation and curation. In the case of the standard of competition, given that it is equally important in online as in traditional media due to concentration being a risk both offline and online, the classical standard was retained, but the methodology to assess it was revised to take into account the specificities of the online media market. In the online sphere, and for the purpose of the media pluralism measurement, the dominance of a certain outlet must be detected through alternative methodologies compared with in traditional media markets. For the purpose of the MPM, symptoms of concentration may be detected by evaluating the competitive advantage of incumbent online platforms when disseminating targeted information based on massive use of personal data and big data. It proved very challenging, nonetheless, to define the benchmarks on pluralism in regard to the new Internet phenomena, considering the ontological diversity of the online environment compared with the traditional media system for which the media pluralism standards have been developed so far, and considering that there is no consensus, even at a policy level, on the solution proposed. As another example, the MPM proposes taxation of online platforms as a method to boost competition on the one hand, and to collect resources to be reinvested in journalism initiatives on the other. However, this is a standard that few EU member states have considered in their media policies.

With this in mind, in the most recent MPM2020 round of monitoring (covering the analysis of the years 2018 and 2019), several new variables related to digital transformation were integrated into the four areas of the MPM (basic protection, market plurality, political independence, and social inclusivity) to obtain a balanced and updated picture of the characteristics of the present media systems in the EU and in selected candidate countries. Furthermore, a special focus on the assessment of digital variables prompted the design of a preliminary evaluation that assesses their specific contribution to the measurement of risks to media pluralism, and that extracts a specific, digital risk score with the intention of advancing the agenda for public discussion and policymaking in the EU (and beyond), focusing on the effects of digital transformation in democratic society.

\section{The results of the MPM2020 round: Conclusions}

The CMPF and the MPM network have finalized the Media Pluralism Monitor 2020 (MPM2020) reports, covering 30 European countries (27 in the EU, along with the UK and two candidate countries, namely Albania and Turkey) (Brogi et al., 2020). The results of the assessment for each area and indicator are presented on a scale from $0 \%$ to $100 \% .^{2}$ The findings show either a general stagnation or deterioration in all four major areas included in the MPM2020.

The MPM2020 confirmed the findings of the previous five rounds ${ }^{3}$ of monitoring, highlighting that none of the analyzed countries are free from the risks of media pluralism. The area of basic protection is assessed at higher risk in comparison with previous rounds, with a score close to being classified as medium risk (33\%), which is quite alarming, considering that this area assesses the fundamental legal requirements for the rule of law in terms of freedom of expression, freedom of information, and conditions of journalists. The average score for the area of market plurality (64\%) is considerably high, signaling the growing economic threats of media pluralism, coming mostly from ownership concentration, in both traditional and digital media markets, and threats to media sustainability. At the same time, the "old" risk relating to commercial and owners' influence over editorial content increased in comparison with previous MPM editions. In the area of political independence, while the risk level (at $47 \%$ ) remained almost the same as in previous rounds, it is remarkable that very few countries are tackling the issue of how to deal with increasingly polarized online political and electoral communication. In the area of social inclusivity, the average score is a $52 \%$ risk, still in the medium risk band.

Going further into the details and general trends in the category of basic protection, the MPM findings confirm that the journalistic profession is facing a deep crisis. In comparison with previous MPM rounds, working conditions for journalists have deteriorated further, exposing them to external and undue pressures within most of the countries examined. Journalists and other media actors continue to face a series of threats and attacks (physical and digital) and are often exposed to strategic lawsuit against public participation (SLAPP) lawsuits. In regard to access to information, the implementation of regulatory safeguarding procedures is still weak in some countries, as well as measures to protect whistle-blowers. In terms of the institutional framework of authorities dealing with the media sector, not all the media regulators can be considered free from political and commercial influences, from the manner of appoint-

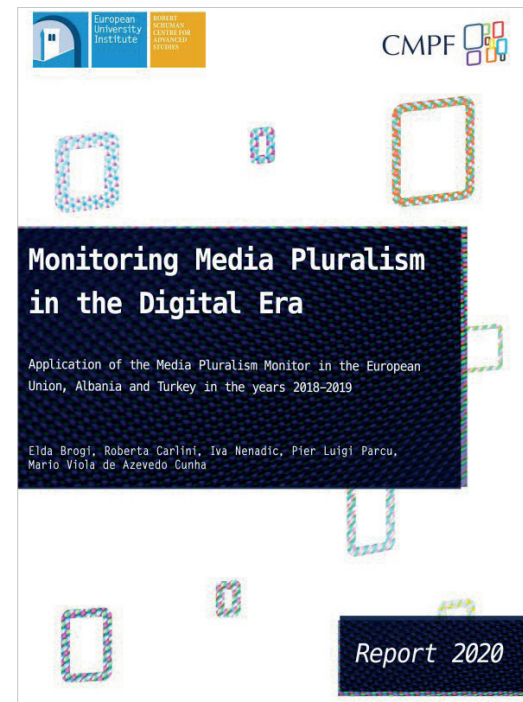

https://hdl.handle.net/1814/67828 
ment of their boards to the implementation of their tasks. As a result, many authorities are not equipped to face the consequences that technological developments will have on policy changes, in terms of both remit and resources.
The "old" risk relating to commercial and owners' influence over editorial content increased in comparison with previous MPM editions

When it comes to market plurality, the monitor reports ineffective legal provisions in terms of media ownership in many states. News media ownership concentration is confirmed to be one of the most significant risks for external pluralism, while the concentration of online platforms is also a high risk across the countries examined. The sustainability of news media is increasingly under threat, as the indicator for media viability registers a high risk in seven countries. The sustainability of new media businesses is impacted by the disruptive role of digital intermediaries that have shifted revenue away from traditional news publishers. Commercial publisher interests interfere with the level of editorial autonomy in many of the countries analyzed. In regard to political independence, news organizations were assessed as being vulnerable to political interference, too. The scarcity of economic resources in media systems raises the potential for political interference, such as the abuse of state advertising being distributed in an unfair, discriminatory, and biased manner. A lack of political independence in public service media, especially in Central and Eastern Europe, is confirmed as a trend in the MPM2020 monitor.

In the area of social inclusivity, lack of gender equality in management and content creation roles, along with a lack of representation for minorities in European media organizations, remains a problem throughout Europe.

What is interesting in the MPM2020 report is the fact that the specific assessment of the digital dimension showed that the digital environment did not evenly contribute to improving media pluralism. In particular, the market plurality and external pluralism areas seemed to be affected by digital disruption. The role of digital intermediaries indirectly affects the viability of the media sector. By collecting users' personal data and using them for targeted advertising, online platforms are the best performers in a market that is competing for the attention of the users. They take a major share of the online advertising market, thus disrupting the traditional business model of news media.

While in the traditional media landscape risks stemmed mainly from the dominance of media outlets at national level, which was easier to regulate (e.g., by media ownership limitations), in today's digital world such dominance by global platforms is much more challenging and difficult to deal with, not only in terms of market dominance. The practices of digital platforms are relevant to assess the levels of freedom of expression; the way they disseminate information even through algorithm-based systems is shaping political and electoral communication, and may have an impact on established democratic procedures, and checks and balances. It is not surprising, therefore, that another interesting result of the MPM is the relatively high score in the risk assessment of fair electoral campaigning online. ${ }^{4}$

As in previous MPM reports, the MPM2020 highlights the lack of data -economic data in particular- as a particular problem. This is particularly evident within the digital landscape, where most of the data relevant for the MPM assessment are currently not collected or not available, sometimes because of the limited transparency of online operators. This may raise a general problem of transparency in itself, to be addressed by policymakers.

Finally, the systematic and holistic approach of the MPM has been considered an asset to be used also for policy purposes. The first rule of law report by the European Commission contains a chapter dedicated to media pluralism and freedom, considered as one of the four axes of the assessment. The MPM results and analysis were used extensively by the general report and by country-specific reports. The MPM, therefore, is now being considered within a policy perspective, with the aim of strengthening the values of democracy, equality, and respect for human rights, all of which form part of the foundations of Europe. This is a recognition of the EU-wide research effort and the investment of many scholars across Europe in the MPM project, as well as an important step towards the establishment of a constructive debate on media pluralism at a European level.

\section{Notes}

1. It must be noted, nonetheless, that in a hybrid media ecosystem, casual exposure to different content remains a likely possibility.

2. Scores between $0 \%$ and $33 \%$ are considered low risk, those from $34 \%$ to $66 \%$ are considered to be medium risk, while those between $67 \%$ and $100 \%$ are considered high risk. In terms of the level of indicators, scores of 0 were rated as $3 \%$ while scores of 100 were rated as $97 \%$, by default, to avoid an assessment of a total absence or certainty of risk, concepts that are in contrast to the natural logic of the MPM tool. 
3. See Brogi et al. (2015; 2016; 2018) and CMPF (2017).

4. The different media ecosystems may produce unforeseen consequences based on the constitutional framework they work in. For example, an electoral campaign that privileges targeted information may unduly influence the results when the margin between the two opposed stances is close, or the use of social media may privilege a political communication that does not allow for a real debate, reshaping the characteristics of some political offices and influencing the form of government.

\section{References}

Brogi, Elda; Carlini, Roberta-Maria; Nenadic, Iva; Parcu, Pier Luigi; Viola-de Azevedo-Cunha, Mario (2020). Monitoring media pluralism in the digital era: application of the Media Pluralism Monitor 2020 in the European Union, Albania \& Turkey. Centre for Media Pluralism and Media Freedom (CMPF), Policy report. https://hdl.handle.net/1814/67828

Brogi, Elda; Dobreva, Alina (2015). Monitoring media pluralism in Europe: testing and implementation of the media pluralism monitor 2014. Centre for Media Pluralism and Media Freedom (CMPF), Policy report. http://hdl.handle.net/1814/38886

Brogi, Elda; Ginsborg, Lisa; Ostling, Alina; Parcu, Pier-Luigi; Simunjak, Maja (2016). Monitoring media pluralism in Europe: testing and implementation of the media pluralism monitor 2015. Centre for Media Pluralism and Media Freedom (CMPF), Policy report.

http://hdl.handle.net/1814/40864

Brogi, Elda; Nenadic, Iva; Parcu, Pier-Luigi; Viola-de-Azevedo-Cunha, Mario (2018). Monitoring media pluralism in Europe: application of the Media Pluralism Monitor 2017 in the European Union, Fyrom, Serbia \& Turkey. Centre for Media Pluralism and Media Freedom (CMPF), Policy Report.

http://hdl.handle.net/1814/60773

CMPF-Centre for Media Pluralism and Media Freedom (2017). Monitoring media pluralism in Europe: application of the media pluralism monitor 2016 in the European Union, Montenegro and Turkey, Policy report.

http://hdl.handle.net/1814/46786

Council of Europe (2018). Recommendation CM/Rec(2018)1[1] of the Committee of Ministers to member States on media pluralism and transparency of media ownership.

https://bit.ly/2TOUFXU

European Commission (2020). Rule of law report. The rule of law situation in the European Union, COM(2020) 580 final. https://ec.europa.eu/info/sites/info/files/communication_2020_rule_of_law_report_en.pdf

Habermas, Jürgen (1995). The structural transformation of the public sphere. An inquiry into a category of bourgeois society. Cambridge, Mass: The MIT Press, 328 pp. ISBN: 9780262081801

Helberger, Natali; Karppinen, Kari; D’Acunto, Lucia (2018). “Exposure diversity as a design principle for recommender systems". Information, communication \& society, v. 21, n. 2, pp. 191-207.

https://doi.org/10.1080/1369118X.2016.1271900

Karppinen, Kari (2013). Rethinking media pluralism. Fordham University Press, 256 pp. https://www.jstor.org/stable/j.ctt13wzz1r

KU Leuven-ICRI (2009). Independent study on indicators for media pluralism - Towards a risk-based approach, European Commission.

https://ec.europa.eu/information_society/media_taskforce/doc/pluralism/pfr_report.pdf

Mill, John-Stuart (1859). On liberty. ISBN: 9780906321638

Moore, Martin; Tambini, Damian (eds.) (2018). Digital dominance: The power of Google, Amazon, Facebook, and Apple. Oxford University Press. ISBN: 9780190845117

Parcu, Pier-Luigi (2019). New digital threats to media pluralism in the information age. EUI RSCAS, 2019/19. Centre for Media Pluralism and Media Freedom (CMPF).

http://hdl.handle.net/1814/61890

Parisier, Eli (2012). The filter bubble: What the internet is hiding from you, Penguin Books: ISBN: 9780143121237 